\title{
LA SUBJECTIVITÉ ÉNONCIATIVE ET LA CONSTITUTION D'IDENTITÉS DANS LE DISCOURS DE LA REVUE FÉMINISTE LA VIE EN ROSE
}

\author{
Marty LAFOREST* \\ Juliane de Araujo GONZAGA **
}

- RÉSUMÉ: La question de l'avortement au Québec suscite encore aujourd'hui des discussions sur sa légalité ainsi que sur le droit des femmes de décider d'avorter. En ce sens, cet article a comme objectif de faire l'analyse d'un éditorial de la revue féministe La vie en rose publié en 1982, en réponse à la lettre antiavortement des évêques, écrite en 1981. À partir des considérations de Kerbrat-Orecchioni concernant l'énonciation et la subjectivité, nous faisons une analyse discursive afin de comprendre comment l'énonciation contribue à la constitution des identités et de saisir comment la subjectivité produit une argumentation dans ces discours. En outre, nous adoptons les notions d'identité de Stuart Hall et de pouvoir de Michel Foucault pour comprendre comment la constitution des sujets dans le langage et dans le contexte socio-historique de production des énoncés rend possible la description des relations de pouvoir exercées entre les féministes, l'Église, l'État et les médecins. Cette analyse nous permet de conclure à la production d'identités multiples pour les femmes, dont l'objectif est de faire résistance face au pouvoir d'institutions hégémoniques.

- MOTS-CLÉS: Avortement. Énonciation. Subjectivité. Identité. Pouvoir.

\section{Introduction}

Le mouvement féministe commence au Québec à la fin du XIXe siècle, initié par les militantes francophones du Conseil national des femmes du Canada. D'une certaine façon, on peut dire que le mouvement au Québec a eu un retard par rapport à celui des Canadiennes anglaises, ce qui peut se comprendre à la lumière de l'engagement des Québécoises dans la religion catholique. De fait, l'Église joue un rôle important dans cette histoire puisque le mouvement, avant de s'imposer de façon plus politique, était conciliable avec la religion catholique. Cette période appelée "féminisme chrétien", où les femmes se sentaient vraiment

\footnotetext{
* UQTR - Université du Québec à Trois Rivières - Département de Lettres et Communcation Sociale. TroisRivières - Québec - Canada. QC G9A 5H7 - marty.laforest@uqtr.ca

** Étudiante à la maîtrise en Linguistique. UFSCAr - Université Fédérale de São Carlos - Département de Lettres. São Carlos - SP - Brésil. 13565-905 - gonzagajuliane@gmail.com
} 
déterminées à prendre des responsabilités inédites dans la société, a été stimulée par le cadre religieux qui a donné aux femmes espace et possibilités pour un développement initial (DUMONT, 2008).

Après des événements importants de l'histoire, comme les guerres mondiales et la crise de 1929, on voit quelques changements culturels qui favorisent des ouvertures pour que les femmes puissent exercer vraiment leurs droits politiques, comme le droit de vote par exemple. Toutefois ce n'était que le début d'une série de luttes qui allaient changer la situation des femmes québécoises. L'une de ces luttes importantes des femmes québécoises a été celle pour le droit à l'avortement libre et gratuit.

L'histoire de l'avortement est caractérisée par son interdiction en 1869 par le Code criminel du Parlement canadien. Cette situation change en 1969, lorsque le gouverment fédéral modifie ce même code et autorise l'avortement thérapeutique. ${ }^{1}$ C'était un projet de loi controversé, parce qu'en même temps qu'il concédait aux femmes le droit d'avorter dans certains cas, il n'empêchait pas la pratique des avortements clandestins ni ne développait des politiques publiques pour améliorer les conditions de pauvreté de familles nombreuses et donner des informations et services de planification des naissances (DESMARAIS, 1999).

Les années soixante-dix sont marquées par un vaste mouvement de contestation sociale et politique, qui refuse l'ordre politique établi en faveur d'un changement dans plusieurs secteurs de la vie quotidienne. Au cours de cette période, plusieurs individus jouent un rôle important dans la résistance aux décisions prises par le gouvernement. L'un de ces personnages a été le docteur Henry Morgentaler, qui offrait des avortements gratuits aux femmes. Dans le prolongement de ce mouvement, on voit, au début des années 1980, la création des centres de santé pour les femmes au Québec, qui fournissaient des informations, des activités d'éducation populaire et des services d'avortement. Il faut remarquer que ces centres n'étaient pas autorisés par les Comités d'avortement thérapeutique, ils travaillaient donc dans l'illégalité (FOPN, 2010).

En 1988, la Cour suprême du Canada exige des modifications à la loi $251 \mathrm{du}$ Code criminel. À partir de ce moment, l'avortement n'est plus considéré comme une pratique illégale, et les femmes peuvent décider librement d'avorter. Il faut mentionner aussi que ce changement a fait que l'avortement au Québec est devenu gratuit et accessible, donc disponible pour les femmes issues de toutes les classes sociales (DESMARAIS, 1999). Durant toute la période qui a précédé

Dans ce contexte, on appelle "avortement thérapeutique" l'avortement qui était fait par un médecin dans un hôpital dès que la femme obtenait une approbation d'un comité responsable qui jugeait si la grossesse impliquait ou non des risques pour sa santé. Il faut remarquer que l'inceste, le viol et la propre volonté d'avorter n'étaient pas des motifs reconnus (DESMARAIS, 1999). 
la légalisation de l'avortement, on voit aussi des actions engagées sur les fronts social et politique, on remarque l'intervention des revues féministes en faveur de l'avortement et leur positionnement contre les mesures et déclarations des évêques, des médecins et des politiciens.

L'une de ces revues féministes est La vie en rose (1982). Cette revue paraît à un moment où le message féministe change de ton, où les besoins des femmes s'expriment sur un ton différent d'autrefois. C'est une prose vivante et décontractée qui se donne à lire dans les pages de La vie en rose, ce qui rend la revue attrayante et en même temps attentive aux événements de l'actualité concernant les femmes. L'un de pricipaux objectifs de cette revue, c'est de rompre avec le ton du féminisme des années 1970 et, surtout, de s'opposer à un féminisme moralisateur et pudique. L'équipe de rédaction est composée par des femmes du Comité de lutte pour l'avortement libre et gratuit, qui animent des débats à l'intérieur du mouvement féministe, qui dénoncent les formes d'oppression et dont le travail se veut attentif au plaisir des lectrices. La vie en rose est l'une de principales revues féministes québécoises et a été publiée pendant huit ans, un record dans la presse féministe (DUMONT, 2008).

En considérant la notion d'énonciation, par laquelle le sujet met en oeuvre le langage et s'approprie son discours, ainsi que ses implications dans la production de la subjectivité, nous souhaitons dans cet article faire l'analyse de l'éditorial "Le droit à la vie?" publié dans La vie en rose en mars 1982 (LE DROIT..., 1982). Nos objectifs sont les suivants: (i) comprendre comment se constituent certaines identités dans et par ce discours; (ii) analyser la constitution de la subjectivité et de l'argumentation et (iii) décrire et comprendre les rapports de pouvoir qui s'exercent dans et par ce discours.

Le texte analysé est une réponse des féministes de La vie en rose à la déclaration antiavortement de l'Assemblée des Évêques de 1981. Ainsi, notre analyse essaiera de faire un parcours depuis l'énonciation jusqu'à l'événement discursif, de façon à intégrer l'acte énonciatif à une mémoire discursive qui produit plusieurs effets de sens dans les discours, les réflexions, les débats d'une époque déterminée de l'histoire du féminisme québécois. Notre analyse adoptera comme démarche "[...] la caractérisation des faits de langue, la connaissance des positions des sujets historiques et de leur conscience linguistique.", c'est-à-dire "[...] la prise en compte de marques énonciatives co-présentes dans l'effet de langue qui constitue l'événement ." (GUILHAUMOU; MALDIDIER; ROBIN, 1994, p.191). On comprend ici "événement" comme un fait qui rompt avec d'autres pratiques discursives et instaure de nouvelles conditions et règles de production du discours. De cette façon, ce travail sera susceptible de contribuer à la connaissance des pratiques discursives rapportées au féminisme et à l'histoire des femmes au Québec. 


\section{Le point de vue de l'énonciation: pour une subjectivité argumentative dans le discours}

L'énonciation se définit comme "[...] cette mise en fonctionnement de la langue par un acte individuel d'utilisation." (BENVENISTE, 1974, p.80). Le travail de l'énonciation est l'action de l'énonciateur pour s'approprier cet appareil formel de la langue et marquer sa position dans le discours. Ce sont des indices spécifiques qui marquent qui parle, à qui l'on parle, quand on parle et d'où l'on parle, ce qui donne une bonne description des composantes qui forment le cadre de la communication.

L'énonciation est responsable de l'instauration d'une situation de communication, où un énonciateur assume la langue et inscrit la présence de l'autre en face de lui. Chaque instance du discours constitue un centre de référence interne, où l'acte individuel d'appropriation du langage introduit celui qui parle et celui à qui l'on parle. C'est la présence de l'énonciateur qui nous fait se rapporter à une classe spécifique de signes, responsables de cet exercice individuel d'insertion de "soi-même" et de l' "autre" dans le discours: c'est le cas des pronoms personnels "je" et "tu" par exemple.

En outre, l'énonciation met en évidence d'autres aspects de la situation de communication, comme l'espace et le temps, qui peuvent être marqués dans le discours par des adverbes et des temps verbaux. Dans le cadre des études de l'énonciation, ces marques sont appelées "déictiques" et sont définies comme des "[...] unités linguistiques dont le fonctionnement sémantico-référentiel [...] implique une prise en considération de certains éléments constitutifs de la situation de communication." (KERBRAT-ORECCHIONI, 1999, p.41).

Dans son ouvrage L'énonciation, Kerbrat-Orecchioni (1999) répertorie quelques déictiques et ajoute quelques observations sur la question des pronoms personnels dans les travaux de Benveniste. La structuration initiale proposée par cet auteur peut être décrite de la façon suivante: "Je" (sujet) parle à un "Tu" (non sujet) se référant à un "Il" (non-personne). Kerbrat-Orecchioni refuse l'idée selon laquelle le "il" assume une fonction de non-personne (ce que croit Benveniste), sauf dans les cas de tournures impersonnelles. D'après la linguiste, il est manifeste que le pronom "il" ne désigne pas spécifiquement rien ni personne mais, si par "en soi" on comprend "hors actualisation", le même phénomène arrive aux pronoms "je" et "tu" qui doivent être insérés dans un cadre communicationnel pour qu'on comprenne leur signification. La différence existant entre ces pronoms, c'est que "[...] généralement, le pronom 'il' a besoin pour recevoir un contenu référentiel précis de déterminations cotextuelles dont le 'je' et le 'tu' peuvent faire l'économie." (KERBRATORECCHIONI, 1999, p.49). 
Kerbrat-Orecchioni développe aussi des réflexions concernant la subjectivité dans le langage, question initialement abordée par Benveniste de façon plus simpliste. Dans le chapitre De la subjectivité dans le langage (1999), la linguiste défend la thèse suivant laquelle la subjectivité se manifeste dans l'ensemble des choix linguistiques et l'organisation des verbes que l'on fait en produisant des énoncés. On remarque que, chez Kerbrat-Orecchioni (1999), il n'y a pas de dichomotomie entre l'énonciation et l'énoncé, au sens où l'énonciation serait l'acte de produire et l'énoncé le produit, le résultat de cet acte. Selon la linguiste, il s'agit du même objet, la différence tenant à la mise en perspective de cet objet. Au surplus, à l'intérieur de ce qui est dit dans l'énoncé, on trouve aussi la présence de l' énonciateur: c'est ce qu'on comprend par l'énonciation.

Pour que l'énonciateur se constitue comme sujet dans et par son énoncé, il faut qu'on envisage le langage comme système de signes de la langue et, en même temps, comme activité manifestée dans les instances du discours. Dans ce procès, il faut qu'on dégage aussi l'aspect pragmatique du langage, de façon à comprendre la constitution du sujet de l'énonciation en fonction de la perspective de l'autre dans le discours. Une fois qu'on énonce "je" ou même son propre nom, s'affirme la conscience de soi dans le discours. Il s'agit d'un positionnement dans l'argumentation, où le sujet se situe en relation avec l'autre, en exprimant son point de vue et ses arguments dans ses énoncés (DEL RÉ, 2009).

Cette réflexion est importante, puisqu'elle suppose que la subjectivité est donnée par l'insertion de l'énonciateur et de l'allocutaire dans le discours, en même temps que sont faits les choix linguistiques lors de la composition de l'énoncé. Pour comprendre les façons dont se constitue la subjectivité dans le langage, on remarque que les unités lexicales sont, en un sens, subjectives, puisque le choix des mots est associé à la représentation que l'énonciateur se fait du monde et du reférent en question. Cela veut dire que, dans le stock d'unités lexicales, il doit en choisir quelques-unes pour verbaliser des objets sur un plan réel ou imaginaire.

On fera attention donc aux types de formulation qu'il choisira. Ces formulations peuvent constituer des discours objectifs (où l'énonciateur s'efforce de gommer son existence) ou subjectifs (où l'énonciateur s'avoue explicitement ou se pose implicitement) (KERBRAT-ORECCHIONI, 1999). La subjectivité s'instaure donc quand l'énonciateur mobilise quelques déictiques ou bien quand il recourt à l'utilisation de quelques adjectifs ou verbes qui dénotent des valeurs affectives ou axiologiques.

Ces considérations nous permettent de conclure que la langue nous offre des éléments pour marquer subjectivement les énoncés et, par conséquent, indiquer à l'allocutaire les directions argumentatives formulées par le énonciateur. La notion d'argumentation suppose l'action d'un énonciateur sur un auditoire, qui vise à modifier ses convictions et à gagner son adhésion (PERELMAN, 2012). De cette 
façon, les marques d'énonciation et la constitution de la subjectivité peuvent exercer des influences mutuelles les unes sur les autres, ce qui leur donne une dimension persuasive.

Selon Amossy (2006, p.31), "[...] le discours argumentatif ne se réduit pas à une série d'opérations logiques et de processus de pensée.", mais " [...] il se construit à partir de la mise en oeuvre des moyens qu' offre le langage au niveau des choix lexicaux qui comportent d'emblée une orientation argumentative." Ainsi, pour produire des énoncés subjectifs et argumentatifs, on doit envisager une approche entre la théorie de l'énonciation et les choix lexicaux dont dispose l'énonciateur.

\section{L'identité et les relations de pouvoir}

Les études culturelles ont commencé à développer la notion d'identité en raison notamment des nouvelles formes d'organisation et de pratiques sociales qu'impose le contexte de la modernité. L'un des principaux effets de la modernité est la crise de l'identité directement liée à une recherche menée par le sujet luimême, qui se demande souvent: "Qui suis-je ?". C'est pourquoi on trouve des travaux qui essaient de comprendre quelles sont les motivations et les principes de la constitution des identités, notamment ceux de Stuart Hall (2008), qui se concentrent surtout dans le champ de la culture et de la politique.

Tout d'abord, on peut définir l'identité comme un phénomène individuel, ce qui veut dire que chaque individu possède une conscience identitaire qui le différencie de tous les autres. La définition de Dorais nous montre que, fondamentalement, l'identité est "[...] la façon dont l'être humain construit son rapport personnel avec l'environnement." (DORAIS, 2004, p.2). La notion d'identité doit donc être expliquée en tant que relationnelle, construite en relation avec l'autre, dans un processus jamais achevé, c'est-à-dire comme une construction établie dans un environnement déterminé où se retrouvent des personnes et des éléments. Autrement dit, si les rapports au monde et à l'histoire changent, l'identité change aussi, suivant les pratiques du sujet avec les autres dans son contexte culturel, politique et social (DORAIS, 2004).

La constitution de l'identité est essentiellement conditionnelle, puisqu'elle a des conditions spécifiques d'existence, ce qui la situe toujours dans la contingence. De la même façon que les autres pratiques signifiantes, l'identité requiert un jeu des différences. Ce processus qui s'opère à travers la différence a plusieurs effets, comme la production de frontières symboliques et de pratiques discursives par exemple. Cela nous donne une conception de l'identité qui n'est pas essentialiste, mais "stratégique et positionnelle". Ainsi le sujet n'est pas supposé comme central et stable, ni non plus comme un sujet qui garantit une "unicité collective" (HALL, 2008). 
D'après cette conception, les identités ne sont jamais unifiées. Au contraire, dans le contexte de la modernité récente, les identités sont de plus en plus fragmentées et fluides. L'identité n'est donc pas l'effet d'une création singulière, mais d'une construction qui l'envisage de façon plurielle dans les discours, les pratiques et les positions sociales. En ce sens, un point important est l'histoire qui détermine les identités en permanence, au sein d'une dynamique toujours en changement, en transformation. Considérer le lien entre l'identité et l'histoire fait nécessairement surgir certaines questions sur l'utilisation de ressources historiques, linguistiques et culturelles dans les processus de constitution des identités. Si l'on pense à partir d'une historisation radicale, la question initiale, "qui suis-je?", s' offre sous un autre jour, et fait place plutôt à des questions comme "qui serai-je?"ou bien "comment serai-je représenté?". Il s'agit d'insérer la constitution des identités à l'intérieur des représentations, qui, selon l'histoire et les pratiques discursives peuvent changer (HALL, 2008).

Il faut remarquer l'importance des pratiques discursives au sein de la constitution de l'identité, puisque les discours sur le sujet participent des formes et modalités du rapport à soi. C'est à partir du "rapport à soi" qu'il est possible de se reconnaître et de se constituer comme sujet, notion que développe Hall (2008), et qui prolonge la théorie des pratiques discursives constitutives du sujet de Michel Foucault (1994b).

Pour Foucault, la constitution du sujet est l'effet des régimes de vérité qui circulent dans notre société. Les régimes de vérité légitiment les savoirs produits par les pratiques discursives et conditionnent l'exercice du pouvoir. Ce sont les conditions qui sont extérieures au sujet qui déterminent de quelle façon il doit se reconnaître et se constituer. Dans les termes foucauldiens, il s'agit plutôt de normes que de conditions, ce qui souligne le lien entre la "constitution du sujet" et les "rapports de pouvoir", de manière à mettre en évidence le dispositif régulateur des pratiques discursives (FOUCAULT, 1994b, p.227).

C'est à travers les normes disponibles qu'advient la "reconnaissance de soi", de sorte que la possibilité d' "être" du sujet est toujours déterminée par un régime de vérité. La conception foucauldienne nous dit qu' “[...] il existe toujours une relation à ce régime, un mode de construction de soi qui se déroule dans le contexte des normes concernées et qui, plus spécifiquement, négocie une réponse à la question de savoir qui le 'je' deviendra en relation à ces normes." (BUTLER, 2007, p.22).

Le problème de la constitution du sujet chez Foucault peut être défini comme la façon dont un sujet fait l'expérience de lui-même dans les jeux de vérité. Cela ne veut pas dire que la constitution du sujet est donnée par une identité psychique, mais à l'intérieur de pratiques de connaissance, de techniques de soi et de rapports de pouvoir, tout en considérant le rôle de l'histoire qui détermine certaines 
modalités. Ainsi, la production historique des subjectivités est initialement liée à un niveau "archéologique", où le travail philosophique trouve et décrit à la fois des savoirs sur le sujet. Après avoir fait la reconnaissance de ces savoirs, les subjectivités sont soumises à une analyse "généalogique" qui fait la description des pratiques de domination et des stratégies de gouvernement dans la constitution du sujet (REVEL, 2005).

Le thème général des recherches de Foucault relève de cette question du sujet. Partout dans ses oeuvres, on trouve aussi une insistance particulière sur la question du pouvoir, qui se justifie par son importance dans la constitution du sujet. D'après l'auteur, le sujet humain est pris dans des rapports de production et des relations de sens autant que dans des relations de pouvoir d'une grande complexité. Il est nécessaire donc de comprendre la notion de pouvoir chez Foucault pour bien saisir son rapport avec la question du sujet.

On peut définir le concept de la façon suivante: le pouvoir n'est pas ce qu'on peut posséder, mais ce qu'on peut exercer. Il ne s'agit pas d'avoir du pouvoir sur les autres, mais d'exercer un pouvoir sur les actions des autres (FOUCAULT, 1994b). Les rapports de pouvoir sont présents dans la vie quotidienne sous plusieurs formes: dans la catégorisation des individus, dans la détermination de leur individualité, dans l'attachement à une identité, dans l'imposition d'une loi de vérité. Selon Foucault, le pouvoir s'exerce à partir de relations entre "partenaires", non pas dans le sens d'un système de jeu entre deux individus, mais simplement par "[...] un ensemble d'actions qui s'induisent et se répondent les unes les autres." (FOUCAULT, 1994b, p.233).

Examiner le thème du pouvoir requiert donc qu'on envisage les relations multiples qui s'exercent dans le cadre social, et non pas un seul pouvoir, dit unilatéral et centralisé. L'idée de pouvoir défendue par Foucault fait référence à de multiples actions développées à la fois, ce sont des relations éparses qui servent à maintenir ou à conditionner de nouvelles normes au sein d'un dispositif de règlements des conduites. Les relations de pouvoir ont comme effet un ensemble d'actions sur des actions possibles, d'où la possibilité d'envisager les comportements des sujets agissants. Le pouvoir est donc susceptible d'inciter, d'induire, de détourner, d'élargir, de limiter, de contraindre ou d'empêcher les actions des sujets.

Après avoir remarqué comment s'exerce le pouvoir, il faut observer aussi l'une des principales conditions de ce "gouvernement" sur les actions des hommes: celle de la liberté. La première condition d'exercice du pouvoir est sans doute qu'on ait des "sujets libres", parce que ce n'est que sur des sujets qui ont pleine liberté qu'on peut exercer quelque type de pouvoir. On comprend par "sujets libres", les sujets individuels ou collectifs qui disposent d'un champ de possibilités où "[...] plusieurs conduites, plusieurs réactions et divers modes de comportement 
peuvent prendre place." (FOUCAULT, 1994b, p.237). Il ne s'agit pas d'une lutte face à face entre le pouvoir et la liberté, mais d'un jeu plus complexe où s'exercent des relations déterminées préalablement par la condition de liberté. Les actions des deux pôles se trouvent ainsi conditionnées par les possibilités et les choix d'actions de sujets libres: il n'y a aucune relation de pouvoir si le sujet ne peut pas décider, en tenant compte de ses possibilités et des jeux de vérités, ce qu'il doit ou non faire.

Une fois qu'on identifie la condition principale du pouvoir, l'examen de cette théorie doit porter attention aussi aux stratégies existantes dans les relations de pouvoir. Si les sujets sont déterminés par les jeux de vérité et si les identités leur sont attachées par les relations de pouvoir, les sujets peuvent bien sûr décider et agir selon les possibilités et représentations dont ils disposent. Cela veut dire que l'assujettissement n'est pas complet, de sorte que le sujet peut trouver des "failles" dans les relations de pouvoir et qu'il peut en profiter au sein d'un processus de constitution de son identité ou de relations qu'il maintient dans les réseaux sociaux.

Cette stratégie appelée "résistance" est fondamentale pour qu'on comprenne la façon dont les relations de pouvoir sont capables de déterminer les "faits de domination", les "révolutions" et les "mouvements de libération" dans les sociétés. C'est l'action de "résistance" qui peut changer un ensemble d'actions et d'exercices de pouvoir au sein d'un état de domination. Pour conclure, on doit remarquer que la résistance se fait "lorsqu'un individu ou un groupe social arrivent à bloquer un champ de relations de pouvoir, à les rendre immobiles et fixes et à empêcher toute réversibilité du mouvement." (FOUCAULT, 1994a, p.711).

\section{L'analyse des marques énonciatives: la construction d'identités et les rapports de pouvoir}

Considérons d'abord le contexte historique où le texte analysé a été écrit. A À la fin des années 1970, le Ministère des affaires sociales (MAS) du Québec annonçait la création de cliniques spécialisées en planification des naissances. Nommées Cliniques Lazure, une référence au ministre qui les avait implantées, ces cliniques fournissaient des services d'avortement thérapeutique, de stérilisation féminine et masculine, et de contraception (FOPN, 2010).

En 1982, année de la publication de l'éditorial analysé, l'avortement était donc déjà possible dans les Cliniques Lazure, mais seulement comme pratique thérapeutique. Il n'existait encore aucune loi qui garantisse le droit aux Québécoises d'interrompre leur grossesse de façon volontaire. À ce momentlà, les parties intéressées - les mouvements féministes, l'État, l'Église et les 
médecins - tentent par tous les moyens d'imposer leurs points de vue respectifs sur la question.

Notre objet d'analyse, l'éditorial "Le droit à la vie?"2 publié dans La vie en rose de mars 1982, fait partie de cet ensemble de manifestations de points de vue, et propose une défense de la pratique de l'avortement volontaire. Il s'agit d'une réponse directe à la déclaration antiavortement de l'Assemblée des Évêques publiée en 1981, déclaration constituant un événement discursif qui a suscité l'émergence des discours pour les droits civils et politiques des femmes. L'éditorial est structuré en arguments et en exemples au sercive d'une prise de position en faveur du droit d'avorter. C'est un texte qui présente le double point de vue défendu d'une part par les militantes féministes et, d'autre part, par l'Église, l'État et les médecins. D'une façon générale, on peut dire que ces discours-là ont permis une rupture avec des discours antérieurs institués sur la question morale et éthique de l'avortement.

Pour analyser cet éditorial, on adoptera une méthodologie qui envisage la description des marques linguistiques rapportées aux sujets impliqués dans ce discours, de façon à comprendre de quelle façon ces marques-là contribuent à la construction de certaines identités. Du point de vue de l'énonciation, on essaiera aussi de vérifier comment l'énonciateur construit une subjectivité en fonction des arguments qu'il produit. Enfin, on fera une analyse des relations de pouvoir exercées entre les sujets, c'est-à-dire qu'on examinera comment s'exercent les relations de pouvoir au sein de la constitution des identités.

Tout d'abord, on observe que l'argumentation développée dans le texte et la constitution des identités sont liées. Cela veut dire qu'en même temps que les choix lexicaux privilégient la construction de certaines identités, ces marques impliquent une direction argumentative, c'est-à-dire qu'elles peuvent être interprétées comme des éléments persuasifs utilisés par l'énonciateur.

Il faut remarquer que l'énonciateur utilise des marques déterminées pour se référer aux personnages impliqués dans le discours. Du point de vue énonciatif, on observe que, dès le début du texte, il s'identifie comme un "nous" et réfère à l'allocutaire au moyen d'un "vous", dont la référence n'est pas identifiée précisement. Par rapport à l'allocutaire, qui sont les récepteurs du message: ce sont seulement des femmes? Seulement des hommes? Le texte ne nous donne pas cette information sous la forme de marques linguistiques, mais en considérant le support en question - une revue féministe - on peut supposer qu'il s'agit plutôt des femmes. En ce sens, le schéma communicatif peut être décrit de la façon suivante: un "nous" (les militantes féministes) qui parle à un "vous" (problablement les lectrices) d'un "ils".

L'éditorial analysé est en annexe à la fin de cet article. 
On arrive ici à un aspect fondamental de la structuration du texte: l'énonciateur "nous" aborde le sujet de l'avortement, mais plus spécifiquement le point de vue d'un "ils" sur l'avortement. Afin de développer son argumentation dans le discours, l'énonciateur "nous" produit des énoncés qui font appel aux conceptions du "ils" sur l'avortement. On peut dire que l'énonciateur "nous" se sert surtout de la stratégie de l'opposion pour se constituer par rapport à "ils". C'est une relation d'altérité qui met en évidence le point de vue de l'énonciateur ainsi que le point de vue du "ils", ce qui, par conséquent, donne une direction argumentative au moyen des marques de subjectivité présentes dans le discours. En ce sens, l'on considère la constitution du sujet comme un effet des représentations ou des images que chacun produit de soi et de l'autre. La constitution du sujet est venue des représentations qui sont parties du "regard de l'autre" (CORACINI, 2010).

Nous refusons la conception du pronom "il" comme "non-personne" proposée par Benveniste (1974). Nous lui préférons la conception de KerbratOrecchioni (1999) selon laquelle le "il", contrairement au "je" et au "tu", a besoin de déterminations cotextuelles. Ce choix théorique se justifie par le fait que, dans le travail de référentiation cotextuelle, le pronom "ils" (c'est bien d'un pluriel qu'il s'agit dans le texte) joue un rôle important dans l'argumentation et dans la constitution de sujets dans le discours. L'aspect constitutif du langage nous permet donc d'avoir une interaction entre les sujets de l'énonciation. Ainsi, on a une relation d'altérité où le "nous" se constitue à mesure qu'on a un "ils" qui est représenté dans et par le langage (CORACINI, 2010).

Dans un premier temps, nous avons donc relevé toutes les marques de désignation dans le discours, c'est-à-dire les marques relatives aux personnes ou aux groupes, et nous avons identifié le référent des pronoms "nous" et "ils". Tout au long du texte, on trouve des marques qui s'assimilent à un "nous" par opposition à un "ils", et d'autres qui sont assimilables à un "ils" en fonction de leur position par rapport au référent "avortement". De là, on comprend donc que s'instaurent deux pôles, deux points de vue différents sur l'avortement. Le tableau ci-dessous présente les différents référents de "nous" et de "ils" relevés dans le texte:

Tableau 1 - Les référents des pronoms "nous" et "ils"

\begin{tabular}{|l|l|}
\hline \multicolumn{1}{|c|}{ PRONOM } & \multicolumn{1}{c|}{ RÉFÉRENT } \\
\hline NOUS & femmes ; journalistes et militantes. \\
\hline ILS & $\begin{array}{l}\text { évêques ; la droite ; théologiens ; médecins ; experts-de-la-vie ; chorale } \\
\text { de bien pensants ; adversaires de l'avortement ; pouvoir patriarcal ; } \\
\text { État et Église. }\end{array}$ \\
\hline
\end{tabular}

Source: Élaboration propre. 
Les référents du "nous" sont assurément des marques plus neutres qui expriment objectivement et positivement ce pôle et qui détermine sa position en faveur de l'avortement. Quant aux référents du "ils", certains renvoient à des réalités objectives comme, par exemple, évêques, État, médecins, la droite, adversaires de l'avortement. D'autres sont nettement subjectifs et peuvent être associées à des valeurs péjoratives, comme par exemple: chorale de bien pensants, experts-de-la-vie et pouvoir patriarcal. Cela nous fait comprendre la construction subjective et même négative du pôle adverse, c'est-à-dire celui qui est contre l'avortement.

L'hypothèse selon laquelle s'affirment deux positions antagonistes dans l'éditorial peut être renforcée par la description des marques verbales. Les marques verbales relatives à l'énonciateur "nous" dénotent assurément une stratégie argumentative où il s'agit de prouver que les femmes du mouvement féministe québécois sont des femmes qui agissent concrètement. Ces verbes contribuent à l'argumentation et à la persuasion, en ce sens qu'il font partie des moyens auxquels l'énonciateur recourt pour faire prévaloir un point de vue favorable à l'avortement. Ainsi, du point de vue sémantique, on remarque que la majorité de verbes relévés expriment une capacité, une action:

(1) des femmes ont manifesté leur crainte d'être culpabilisées encore plus dans les jours suivants;

(2) nous parlons de vie pour éviter de parler de morte;

(3) nous nous sommes réunies pour concocter un projet de revue féministe d'information;

(4) nous avions travaillé quotidiennement au Comité de lutte pour l'avortement libre et gratuit;

(5) nous avions fait de la référence;

(6) nous avions animé des soirées d'information;

(7) nous avions assisté à des avortements;

(8) dès le début de notre lutte pour exiger l'accessibilité á l'avortement;

(9) nous prenons le droit de le faire;

(10) nous appuyons toute femme qui a besoin d'être appuyée.

Du point de vue sémantique, les verbes relevés font référence aux actions et aux efforts entrepris par les militantes dans la lutte pour l'avortement. Si l'on prend en compte ces sens-là et le contexte de l'énonciation, on a une situation pragmatique qui nous permet d'envisager un procès de persuasion, où l'énonciateur fait prévaloir une représentation des militantes féministes engagées 
dans la cause de l'avortement. L'emploi de verbes d'action imprime des sens favorables aux idées de lutte pour l'avortement; de décisions pour leur propre corps; de résistance face aux intérêts de l'hégémonie et de changement du cadre existant à cette époque-là. En considérant donc le contexte d'énonciation, les sens d'action et de capacité, et le mode verbal indicatif, on peut dire qu'il s'agit d'un discours qui produit une argumentation en faveur d'une représentation de militantes actives imposant leurs revendications.

Par ailleurs, on trouve d'autres marques verbales relatives au "nous", qui font appel aux émotions. Comparés à la catégorie sémantique d'action et de capacité, les verbes associés aux émotions sont moins fréquents tout au long du texte. Il faut remarquer que l'emploi de ce type de verbe a aussi pour but de persuader l'allocutaire, puisque les émotions jouent un rôle important dans le procès d'adhésion aux arguments défendus par l'énonciateur (PERELMAN, 2012).

(11) nous répugnions sans doute à ajouter notre voix à cette chorale de bien pensants;

(12) décide de se choisir elle-même et de sacrifier l'autre;

(13) nous ressentions alors - et encore aujourd'hui - à entendre théologiens, médecins, et autre experts de-la-vie;

(14) nous avons tu la question même entre nous;

(15) Que nous risquions un peu plus ou un peu moins notre santé ou notre vie, cette vie-là ne les intéresse pas;

(16) Si nous n'étions pas si vulnérables à leur entreprise de culpabilisation et de contrôle, leur discours grossier nous ferait crouler de rire.

En plus, ces marques verbales renvoient à des pratiques discursives attribuées culturellement et historiquement aux femmes. Ce sont des marques qui dénoncent des pratiques et qui, par conséquent, représentent des arguments favorables à la lutte des militantes féministes. Les verbes nous font donc saisir un travail d'argumentation qui repose sur l'exposé d'un ensemble de pratiques discursives associées aux femmes. En présentant des exemples et en faisant appel aux sentiments, l'énonciateur construit un texte dont le but principal est d'émouvoir et de convaincre l'allocutaire qu'il faut changer les pratiques sociales et donner aux femmes le droit de décider de leur corps.

On peut remarquer aussi l'occurrence de nombreuses formes passives du verbe, qui contribuent à construire une représentation des femmes comme des sujets fragiles, même des victimes, comme le montrent les exemples suivants.

(21) la question de la vie et de la mort, nous a harcelées sans que nous n'osions en parler; 
(22) ce geste a été interdit, toléré, et parfois imposé;

(23) c'est une rupture fondamentale avec tout ce qui nous a été inculqué.

Quant aux marques verbales associées au pôle adverse, on trouve là encore des occurrences de verbes vraiment subjectifs, qui font appel aux émotions ainsi qu'à une idée de supériorité (exemples 24 à 30).

(24) à entendre théologiens, médecins et autre experts-de-la-vie s'arroger le droit de dicter la vérité;

(25) Les États et les Églises se sont de tous temps arroger le droit à tuer, voire même de massacrer au nom de l'intérêt national;

(26) Que n'ont-ils pas fait pour conquérir un territoire?

(27) nous avons toujours soingneusement contourné le débat moral où la droite voulait nous confiner;

(28) Quand les évêques haussent le ton et semoncent le gouvernement pour ses cliniques Lazure, ils ne se gênent pas pour affirmer "qu'un avortement est un avortement fait à l'hôpital ou dans la clandestinité...";

(29) les évêques condamnaient une fois de plus l'avortement comme un geste meurtrier;

(30) Profitant de notre silence, elle (la droite) a monopolisé jusqu'ici tout le terrain.

Toutefois, les verbes associés au "ils" ne sont pas aussi nombreux que ceux associés au "nous". Dans l'éditorial analysé, 78 verbes sont associés au "nous" et seulement 39 verbes au "ils". Le "nous" se constitue par les choix et l'emploi de verbes comme un sujet à la fois victime et en lutte par l'action contre la sujétion qu'il subit. Par ailleurs, le "ils" se constitue comme sujet dans une position hégémonique, qui préconisent des mesures peu favorables pour les femmes. Ainsi, on peut dire que ces choix verbaux donnent une direction argumentative au discours, de manière à légitimer une représentation négative du "ils" et à convaincre le lecteur qu'il s'agit d'un groupe hostile aux femmes.

Dans l'éditorial analysé, on comprend que la relation du "nous" avec le "ils" met en scène une relation d'altérité, où l'interaction d'un "je" avec l'“autre"constitue les sujets de l'énonciation. Au fur et à mesure que le "je" s'assume comme énonciateur de son discours, on a l'instauration énonciative du récepteur et du référent du message. Ce type de relation se trouve dans le langage ainsi que dans la vie sociale, ce qui nous fait penser à l'aspect constitutif du langage. Le langage fait partie de la société et, à la fois, estdéterminé et modifié par elle. En ce sens, il faut qu'on comprenne l'altérité comme un procès linguistique et social, puisque ce sont les relations dans le langage et dans la 
société qui constituent les identités. Lorsque le sujet joue des rôles déterminés dans le langage et dans les relations sociales, il devient conscient de soi et des autres (CORACINI, 2010).

Puisque les pratiques sociales influencent la constitution des identités, il faut que l'on envisage le langage comme médiateur de l'action sociale. Ainsi, à partir des marques énonciatives de constitution des sujets et de l'instauration de la subjectivité, nous avons saisi certaines identités issues du discours analysé.

Dans la perspective de Hall (2006), l'identité est résultante des relations entreprises dans le langage ainsi que dans la culture et l'histoire d'un groupe déterminé. Le travail de description linguistique fait conclure que les marques énonciatives représentent des pratiques discursives et, par conséquent, participent du cadre historique en question. La désignation des personnages du texte ainsi que les choix verbaux mettent en évidence les conditions et les règles déterminant la production des discours féministes au Québec, dans les années 1980. Cela nous permet d'inférer les pratiques discursives propres à cette époque et de dégager la circulation de discours associés aux femmes et au pouvoir patriarcal.

Dans l'histoire du Québec, le mouvement des femmes a été l'acteur le plus important dans le cadre de représentation politique féminine. Les efforts du mouvement ont contribué pour les gains qu'on fait les femmes au cours des 30 dernières années. En ce sens, on remarque le rôle joué par les vagues du féminisme. La première vague correspond au moment où les femmes revendiquaient des droits dans la sphère publique, comme par exemple le droit de vote et le droit d'être elues. La deuxième vague du féminisme revendique aussi de droits pour les femmes, surtout ceux qui sont associés à la sphère privée, comme le droit de disposer de son propre corps, d'avorter libre et gratuitement, aussi que le droit à l'égalite et par extension à la parité (TRUDEL, 2009).

L'histoire rappelle qu'a prévalu une situation de marginalisation des femmes résultant de contraintes morales et religieuses dans la société occidentale. L'ensemble des règles et disciplines qui régulent les "vérités" sur le corps féminin n'ont pas permis un usage du corps pour le plaisir féminin, mais seulement pour la procréation. Pendant longtemps, le sexe n'était qu'une façon de perpétuer la race humaine, et cette détermination était inspirée surtout par l'Église catholique et les efforts de surveillance et discipline de l'État (FOUCAULT, 1976).

L'Église, l'État et la médecine au Québec occupaient une position dominante, ce qui rendait possible la production de discours interdisant l'avortement au profit de la procréation. La position occupée dans l'histoire par les militantes féministes limitait celles-ci dans leur manifestation, les conditions n'étaient pas réunies pour une expression forte et libre. Toutefois, si l'on fait attention au lieu occupé par 
l'énonciateur de l'article analysé - une revue féministe qui défendait les droits politiques des femmes dans les années 1980 - on peut trouver des éléments plus favorables à l'émergence de discours qui défendent l'avortement et le droit de décider de son propre corps.

À l'époque de production de l'éditorial, le premier ministre québécois était René Levesque, président du Parti Québécois. L’organisation politique du Québec était donc fondée sur un parti "démocratique" en opposition à celui libéral, qui ressemblait fort à celle des États-Unis et des plusieurs provinces canadiennes. Par contre, même le gouvernement démocratique trouvait des critiques parmi les féministes à cause de ses mesures qui renvoyaient le pays à un plan conservateur. Tout en affirmant une posture "pas partisane" la Féderation feministe du Québec "[...] encourage les femmes à aller voter et à faire valoir leurs attentes face au gouvernement." (TRUDEL, 2009, p.321). Àcette époque la scène fédérale a vu des revendications dans les secteurs les plus divers de la société québécoise. Il s'agissaient des demandes concernant

[...] le rôle de l'État dans la redistribution de la richesse collective, la lutte à la pauvreté, la réinjection des fonds dans les services publics, l'augmentation du salaire minimum, des mesures d'aide aux femmes des communautés culturelles, aux non-syndiquées, et pour améliorer le régime d'assurance parentale, des congés pour les personnes qui prennent des proches en charge et des services d'avortement accessibles dans toutes les régions. (TRUDEL, 2009, p.321).

En considérant le contexte de tranformation sociale au Québec au cours des années 1980 - l'émergence de discours contre les tentatives de domination religieuse (DUMONT, 2008) et les revendications sociales pour meilleurs conditions de vie (TRUDEL, 2009) - on voit le début du changement des "jeux de vérités" qui régulent la constitution de certaines identités dans le discours analysé. L'énonciateur dispose d'une certaine liberté pour s'exprimer et pour opposer une résistance au pouvoir exercé par l'Église et l'État, par exemple. Pour comprendre la relation entre le pôle des militantes (celui du "nous") et le pôle adverse (celui du "ils"), on peut rappeler quelques énoncés déjà présentés plus haut:

(21) la question de la vie et de la mort, nous a harcelées sans que nous n'osions en parler;

(22) ce geste a été interdit, toléré, et parfois imposé;

(23) c'est une rupture fondamentale avec tout ce qui nous a été inculqué;

(28) Quand les évêques haussent le ton et semoncent le gouvernement pour ses cliniques Lazure, ils ne se gênent pas pour affirmer "qu'un avortement est un avortement fait à l'hôpital ou dans la clandestinité..."; 
(29) les évêques condamnaient une fois de plus l'avortement comme un geste meurtrier;

(30) Profitant de notre silence, elle (la droite) a monopolisé jusqu'ici tout le terrain.

Les "failles" du discours nous font nous interroger sur ce qui est dit, pour les analyser comme des effets discursifs de la relation existant entre les femmes et le pôle adverse. Dans les exemples (21), (22) et (23), on trouve des marques linguistiques passives qui font que l'énonciateur se constitue en "victime". En outre, en analysant les exemples (28), (29) et (30), on constate que se tisse tout un réseau de relations de pouvoir dont l'effet est d'instituer le pôle adverse comme "dominateur" ou même comme "agresseur". On dénonce ainsi toute une histoire de domination fondée sur des pratiques et des discours associés au corps féminin, de façon à le marginaliser, lui interdire le plaisir et l'instituer comme appareil reproducteur.

On trouve dans les études féministes la notion de genre comme catégorie d'analyse historique. Cela veut dire que les relations entre les femmes et les hommes sont perçues tout au long de l'histoire de façon à signifer les relations de pouvoir. Selon Scott (1995), le genre est élément constituant les relations sociales fondées sur les différences entre les sexes. Les différences sexuelles contribuent pour l'exercice des relations de pouvoir, qui resultent des savoirs produits et partagés au sein des cultures et des sociétés, et qui sont utilisés dans les situations de domination et subordination entre les genres. En ce sens, la notion de genre comme catégorie d'analyse historique permettent de décrire et de comprendre les discours rapportés aux différences sexuelles et aux processus politiques qui catégorisent l'homme et la femme (SCOTT, 1995).

En même temps, le discours analysé continue à construire l'identité des femmes, puisque la constitution de l'identité n'est ni un procès achevé, ni un procès homogène. D'après Hall (2006), le sujet peut être pensé comme fragmenté, constitué par plusieurs identités contraditoires et indéterminées. Cela veut dire que, sans se limiter à la constituer comme "victime", le discours analysé présente aussi une identité de "militantes", une identité de femmes qui luttent pour la cause de l'avortement et de la liberté de leurs corps. C'est ce qu'on peut voir dans les exemples suivants:

(3) nous nous sommes réunies pour concocter un projet de revue féministe d'information;

(4) nous avions travaillé quotidiennement au Comité de lutte pour l'avortement libre et gratuit;

(5) nous avions fait de la référence;

(6) nous avions animé des soirées d'information; 
(7) nous avions assisté à des avortements;

(9) nous prenons le droit de le faire;

(10) nous appuyons toute femme qui a besoin d'être appuyée.

L'analyse fait donc apparaître la constitution d'une double identité de l'énonciateur comme "victime" et "militante". Nous justifions cette proposition par les énoncés qui mettent en évidence la relation de pouvoir entre les femmes et le pouvoir patriarcal. Cette conclusion repose sur le fait que le énonciateur attribue aux femmes des actions et capacités propre à un mouvement qui exige des modifications dans la loi et dans les pratiques sociales. Cela se justifie aussi par les transformations du cadre historique du Québec dans les années 1980: c'est une époque de contestation contre le pouvoir de l'Église et même contre quelques mesures du gouvernement. Les transformations de la société changent aussi les identités, qui deviennent fragmentées et inachevées.

De plus, le discours analysé nous fait saisir les procès d'objectification, de normalisation et de disciplination des sujets. Le discours de la médecine, de l'Église et de l'État construisent des vérités dont la finalité est d'exercer leur pouvoir sur et dans le corps des sujets. En ce sens-là, on comprend le corps comme instrument et espace des relations de pouvoir. Ainsi, le corps objectivé n'a qu'une subjectivité à partir du moment où il lui est attribué une sexualité. Le pouvoir exercé par l'Église, l'État et la médecine s'étend dans toute la société, de façon à contrôler, à surveiller et à discipliner le corps. De ce procès d'objectification et subjectification, on prend la stratégie principale du pouvoir sur les corps: les corps participent aux organisations sociales de façon rigoureuse et contrôlée, et, en même temps, représentent un moyen pour que le sujet constitue leur propre sexualité.

Au Québec, le contexte de luttes entre les féministes, l'Église, l'État et les médecins dès les années 1960, où l'on commence à discuter l'avortement dans le Code criminel. En 1975, l'avortement est encore un crime, toutefois, certains groupes de femmes se sont organisés et ont formé le Comité de lutte pour l'avortement et la conception libres. Le comité revendique la décriminalisation de l'avortement de façon polémique, vu qu'elles sont allées au médias pour exposer des problèmes qui leur arrivent et faire quelques demandes au gouvernement comme: la création de garderies, le droit au salaire égal, l'avortement, la lutte au sexisme, l'entrée des femmes dans la politique. Le mouvement féministe arrive aux publicités, paraisse dans des journaux et parle aux femmes québécoises directement (TRUDEL, 2009).

Toutefois, l'opposition des féministes est mise contre l'État et ses mesures, ainsi que contre l'Église. La lettre antiavortement des évêques, écrite en 1981, met en scène la relation entre l'Église et les féministes, vu qu'il s'agit d'une lettre 
dont les propositions s'opposent à celles du féminisme québécois. Pour l'Église, l'avortement n'est pas uniquement un problème à la femme, il s'agit aussi d'un problème masculin, le père a autant des responsabilités que la mère. L'Église renforce aussi l'argument selon lequel l'avortement ne peut pas changer ni non plus améliorer la situation de la femme. La libération de la femme, en ce sens-là, ne peut pas être liée à l'avortement si elle est obtenue "au détriment d'un autre être humain". Les déclarations de l'Église à l'époque remarquent de la même façon le rôle de l'État: il faut faire attention au bien commum de la société et faire des efforts pour une meilleure qualité de la vie. Ils deffendent donc que toute interdiction à l'avortement est une façon de maintenir et contrôler le bien commun des citoyens (ASSEMBLÉE DES ÉVÊQUES DU QUÉBEC, 1981).

Notre analyse comprend donc le discours de La vie en rose comme un discours où les sujets se constituent linguistiquement et historiquement. Le but de la constitution du sujet est donc en l'occurrence de résister au pouvoir exercé par le pôle adverse, qui a triomphé pendant longtemps à cause de sa position politique hégémonique dans l'histoire québécoise. Dans la contemporaneité, on voit que la question sur l'avortement est encore problématique et suscite beaucoup de discussions entre les politiques et le représentants religieux. En 2008, par exemple, lors des éléctions fédérales au Canada, l'évêques se sont mis à parler de l'avortement dans le but de protester contre les partis qui se montrent pour l'avortement, du même pour le titre d'honorifique au Docteur Morgentaler (defenseur de l'avortement) (MAISONNEUVE, 2008). Si l'on pense aux relations de pouvoir en tant qu'elles se diffusent et s'exercent au sein de tout le réseau social, on peut conclure que la production de cet éditorial, ainsi que la constitution des identités de "victimes", de "militantes"et d' "agresseurs", font partie des luttes et des relations de pouvoir existant entre les femmes, l'Église, l'État et les médecins au Québec.

\section{Conclusion}

À partir de la notion d'énonciation selon laquelle le sujet met en fonctionnement le langage et s'introduit dans son discours, en produisant la subjectivité dans le langage, cet article a proposé une analyse de l'éditorial "Le droit à la vie?"publié dans La vie en rose en mars 1982. Nous avons dégagé les principales marques énonciatives qui instaurent la subjectivité et, par conséquent, construisent quelques identités dans et par le langage. Le choix de substantifs pour nommer les personnages du discours ainsi que les marques verbales, nous ont fait comprendre que le texte analysé construit une argumentation en faveur du droit d'avorter.

Le schéma communicatif analysé nous a permis de comprendre la stratégie discursive choisie par l'énonciateur: se constituer à partir de points de vue 
argumentés. Ainsi on a un "nous" dont le point de vue se constitue par opposition à celui d'un "ils". De cette façon, les choix lexicaux ont privilégié la constitution d'identités fluides, comme dans le cas des femmes, à la fois "victimes" et "militantes", et d'identités attribuées aux adversaires, comme "agresseurs" et "dominateurs". À partir de l'analyse linguistique de ces marques énonciatives, et de quelques informations sur le contexte historique entourant la question de l'avortement au Québec, nous avons pu constater les relations de pouvoir qui s'exercent, où le pôle dominant est celui que forment l'Église, l'État et les médecins. La position de "dominées", toutefois, est contestée par cet éditorial dont la position en tant que sujet permet à l'énonciateur de produire des discours comme forme de résistance.

Si l'on pense le langage comme un médiateur des relations existant dans la société, on peut comprendre la constitution d'identités comme un procès d'altérité où le "je" est toujours en relation avec l'"autre". Ainsi, l'histoire, l'interaction entre les sujets et les pratiques discursives circulant dans la société jouent un rôle fondamental dans cette constitution d'identités. Cet article peut aider à comprendre les procès d'une telle constitution ainsi que les relations de pouvoir entre l'Église, l'État, les médecins et les femmes qui pratiquent l'avortement. L'analyse du discours et de l'histoire nous ont donc permis de replacer l'éditorial de La vie en rose dans son contexte extralinguistique et de comprendre comment les lieux historiques et sociaux occupés par chaque identité sont importants pour la production discursive. Ce faisant, nous croyons pouvoir donner un éclairage nouveau à la question des pratiques discursives qui caractérisent les luttes pour les droits des femmes du Québec.

\section{Remerciements}

Tout d'abord, nous remercions le Ministère des Affaires Étrangères du Canada qui a financé le développement de cette recherche. Nous remercions également le Professeur Marc-André Bernier, qui nous a beaucoup aidées avec la révision grammaticale du texte, et la traductrice Thaís Tamaoki qui a traduit le résumé en anglais.

LAFOREST, M.; GONZAGA, J. deA. The enunciative subjectivity and the constitution of identities in the discourse of feminist magazine La vie en rose. Alfa, São Paulo, v.58, n.2, p.323-346, 2014.

- ABSTRACT: The question of abortion in Quebec still gives rise to discussions about its legality and women's right to choose. In this sense, this article aims to analyze the editorial of the feminist magazine La Vie en Rose, published in 1982, as a response to the anti-abortion letter written in 1981 by bishops. Based on considerations on enunciation and subjectivity by Kerbrat-Orecchioni, a discursive analyze will be made in order to explain how enunciation 
contributes in the formation of identities and how subjectivity generates arguments in such discourses. In addition, the notion of "identity"formulated by Stuart Hall and the notion of "power", by Michel Foucault, will be used to explain how discursive constitution of identities in language and in the socio-historical context of discursive production enables the description of relations of power between feminists, the Church, the State and doctors. This analysis will verify the production of multiple identities for women whose goal is to make resistance to the power of hegemonic institutions in Quebec.

- KEYWORDS: Abortion. Enunciation. Subjectivity. Identity. Power.

\section{RÉFÉRENCES}

AMOSSY, R. L'argumentation dans le discours. Paris: Armand Colin, 2006.

ASSEMBLÉE DES ÉVÊQUES DU QUÉBEC. Un appel en faveur de la vie. Montréal. 1981. Disponible sur le site:<http://www.eveques.qc.ca/documents/1981/1981129f. html>. Date de lecture: 23 juil. 2013.

BENVENISTE, E. L'appareil formel de l'énonciation. In: Problèmes de Linguistique Générale II. Paris: Éditions Gallimard, 1974. p.79-88.

BUTLER, J. Le récit de soi. Paris: PUF, 2007.

CORACINI, M. J. Transdisciplinaridade e análise do discurso: migrantes em situação de rua. Cadernos de Linguagem e Sociedade, Brasília, v.11, n.1, p.91-112, janv. 2010. Disponible sur le site: <http://seer.bce.unb.br/index.php/les/article/ view/1181/844>. Date de lecture: 22 janv. 2013.

DEL RÉ, A. Um caminho em direção à constituição da identidade na criança: enunciação, linguagem e cognição. Letras de Hoje, Porto Alegre, v.44, n.3, p.4452, juil./sept. 2009. Disponible sur le site: <http://revistaseletronicas.pucrs.br/ ojs/index.php/fale/article/viewFile/5763/4183> . Date de lecture: 22 janv. 2013.

DESMARAIS, L. Mémoires d'une bataille inachevée: la lutte pour l'avortement au Québec. Montréal: Éditions Trait d'union, 1999.

DORAIS, L. J. La construction de l'identité. In: DESHAIES, D.; VINCENT, D. (Org.). Discours et constructions identitaires. Québec: Les presses de l'université Laval, 2004. p.1-10.

DUMONT, M. Le féminisme québécoise raconté à Camille. Montréal: Les Éditions du remue-ménage, 2008.

FÉDÉRATION DU QUÉBEC POUR LE PLANNING DES NAISSANCES [FOPN]. Le point sur les services d'avortement au Québec. 2010. Disponible sur le site: < http://www.foqpn.qc.ca/?attachment_id=1287>. Date de lecture: 21 janv. 2013. 
FOUCAULT, M. L'éthique du souci de soi comme pratique de la liberté. In:

Dits et écrits IV. Paris: Éditions Gallimard, 1994a. p.708-729.

. Le sujet et le pouvoir. In: Dits et écrits IV. Paris: Éditions Gallimard, 1994b. p.222-243.

L'hypothèse répressive. In: Histoire de la sexualité I: la volonté de savoir. Paris: Éditions Gallimard, 1976. p.23-67.

GUILHAUMOU, J.; MALDIDIER, D.; ROBIN, R. De l'énonciation à l'événement discursif en analyse du discours. In: . Discours et archive. Liège: Mardaga, 1994. p.185-192.

HALL, S. Qui a besoin de l'“identité"? In: Identités et cultures: politiques des cultural studies. Paris: Éditions Amsterdam, 2008. p.267-285.

2006.

. A identidade cultural na pós-modernidade. 11e. éd. Rio de Janeiro: DP\&A,

KERBRAT-ORECCHIONI, C. L'énonciation. Paris: Armand Colin, 1999.

LE DROIT à la vie? La vie en rose, Montréal, n.5, p.4-5, mars 1982.

MAISONNEUVE, P. L'avortement, les évêques et la politique. Radio Canada, 11 sept. 2008. Disponible sur le site: <http://www.radio-canada.ca/radio/ maisonneuve/11092008/105734.shtml>. Date de lecture: 23 juil. 2013.

PERELMAN, C. L'empire rhétorique. Paris:Vrin, 2012.

REVEL, J. Expériences de la pensée: Michel Foucault. Paris: Bordas, 2005.

SCOTT, J. Gênero: uma categoria útil de análise histórica. Educação e Realidade, Porto Alegre, v.20, n.2, p.71-99, 1995.

TRUDEL, F. L'engagement des femmes en politiques au Québec: histoire de la Fédération des femmes du Québec de 1966 à nos jours. 2009. 510f. Thèse (Doctorat en Histoire) - Université du Québec à Montréal, Montréal, 2009. Disponible sur le site: <http://www.archipel.uqam.ca/2212/1/D1814.pdf>. Date de lecture: 23 juil. 2013. 


\section{Annexe}

Éditorial "Le droit à la vie?2". La vie en rose, Montréal, n. 5, p.4-5, mars 1982.

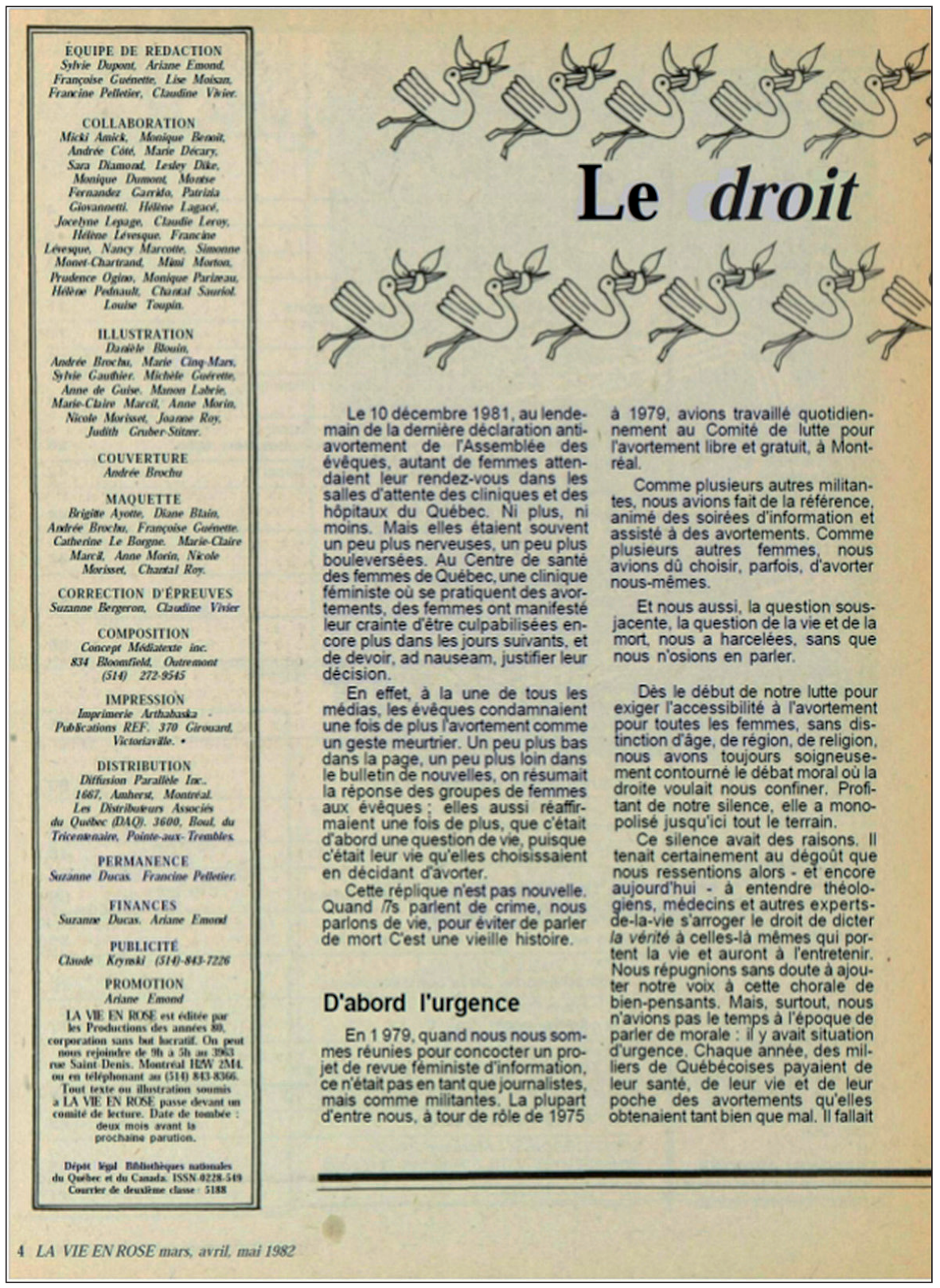




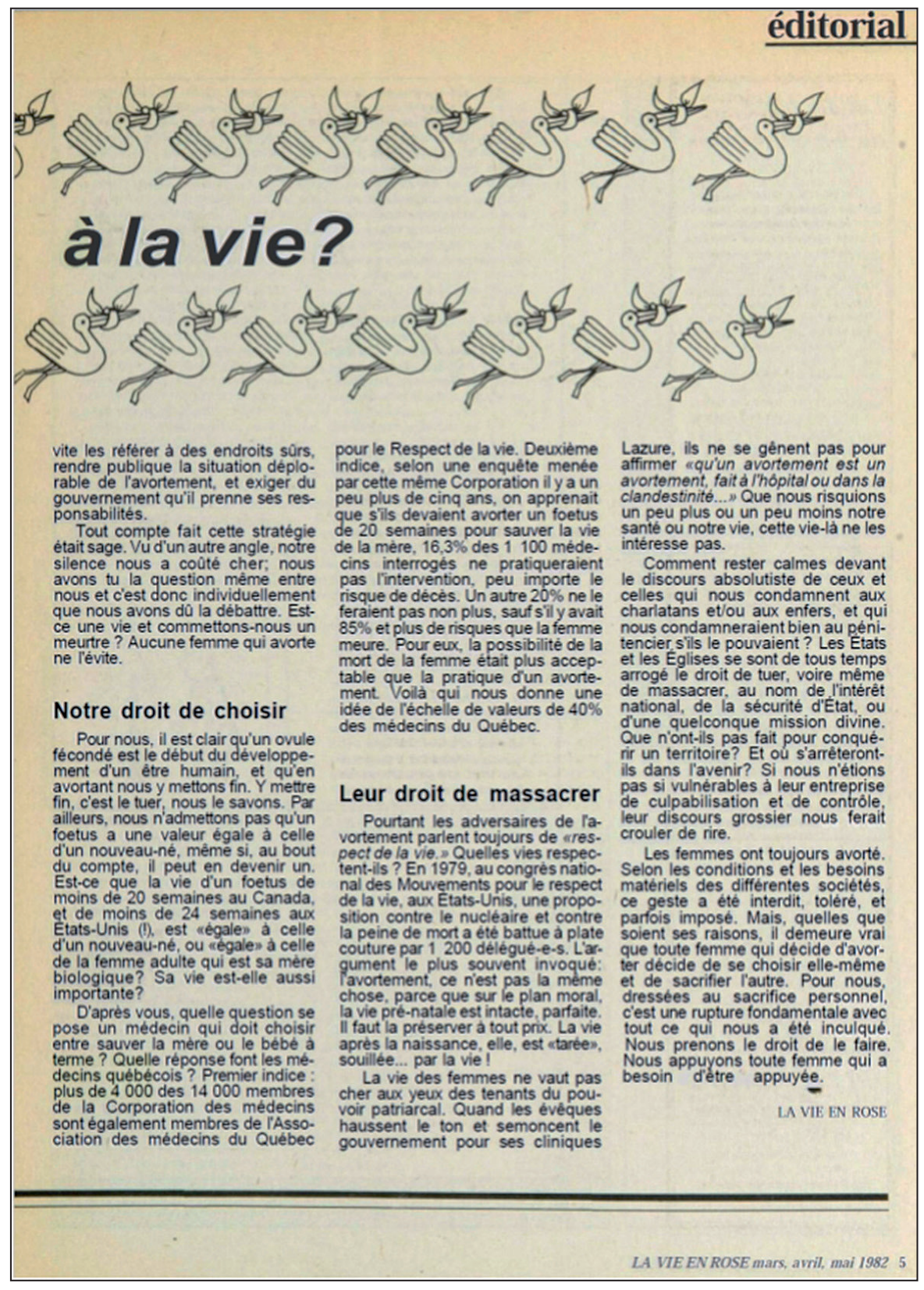

Recebido em fevereiro de 2013.

Aprovado em agosto de 2013. 